\title{
Reviewers for Kew Bulletin Volume 70
}

We are grateful to the following people who reviewed articles for Kew Bulletin during the years $2014-2015$.
R. Agerer
J. Beck
P. F. Cannon
L. G. Clark
A. P. Fortuna-Perez
V. A. Funk
R. E. Gereau
D. A. German
A. M. Giulietti Harley
P. Goldblatt
R. E. Halling
W. D. Hawthorne
B. R. Jackes
J. Kalema
M. Kato
R. Kiew
J. H. Kirkbride
I. Larridon
C. Lindqvist
A. McDonald

D. Medan

D. J. Middleton

C. M. Morton

A. M. Muasya

P. O'Byrne

S. Ortiz

J. F. B. Pastore

G. T. Prance

R. M. K. Saunders

M. S. M. Sosef

C. A. Stace

M. Thulin

J. R. Timberlake

E. Tripp

N. J. Turland

I. M. Turner

D. C. Wasshausen

M. J. Waterway

P. Wilkie

J. R. I. Wood 